\title{
Modeling clustered activity increase in amyloid- beta positron emission tomographic images with statistical descriptors
}

\author{
Sepideh Shokouhi' \\ Baxter P Rogers' \\ Hakmook Kang ${ }^{2}$ \\ Zhaohua Ding' \\ Daniel O Claassen ${ }^{3}$ \\ John W Mckay' \\ William R Riddle' \\ On behalf of the \\ Alzheimer's Disease \\ Neuroimaging Initiative \\ 'Department of Radiology and \\ Radiological Sciences, Vanderbilt \\ University Institute of Imaging \\ Science, ${ }^{2}$ Department of Biostatistics, \\ ${ }^{3}$ Department of Neurology, Vanderbilt \\ University, Nashville, TN, USA
}

This article was published in the following Dove Press journal:

Clinical Interventions in Aging

20 April 2015

Number of times this article has been viewed

Background: Amyloid-beta (A $\beta$ ) imaging with positron emission tomography (PET) holds promise for detecting the presence of $A \beta$ plaques in the cortical gray matter. Many image analyses focus on regional average measurements of tracer activity distribution; however, considerable additional information is available in the images. Metrics that describe the statistical properties of images, such as the two-point correlation function $\left(\mathrm{S}_{2}\right)$, have found wide applications in astronomy and materials science. $\mathrm{S}_{2}$ provides a detailed characterization of spatial patterns in images typically referred to as clustering or flocculence. The objective of this study was to translate the two-point correlation method into A $\beta$-PET of the human brain using 11C-Pittsburgh compound $\mathrm{B}(11 \mathrm{C}-\mathrm{PiB})$ to characterize longitudinal changes in the tracer distribution that may reflect changes in $\mathrm{A} \beta$ plaque accumulation.

Methods: We modified the conventional $\mathrm{S}_{2}$ metric, which is primarily used for binary images and formulated a weighted two-point correlation function $\left(\mathrm{wS}_{2}\right)$ to describe nonbinary, realvalued PET images with a single statistical function. Using serial 11C-PiB scans, we calculated $\mathrm{wS}_{2}$ functions from two-dimensional PET images of different cortical regions as well as three-dimensional data from the whole brain. The area under the $\mathrm{wS}_{2}$ functions was calculated and compared with the mean/median of the standardized uptake value ratio (SUVR). For threedimensional data, we compared the area under the $\mathrm{wS}_{2}$ curves with the subjects' cerebrospinal fluid measures.

Results: Overall, the longitudinal changes in $\mathrm{wS}_{2}$ correlated with the increase in mean SUVR but showed lower variance. The whole brain results showed a higher inverse correlation between the cerebrospinal $\mathrm{A} \beta$ and $\mathrm{wS}_{2}$ than between the cerebrospinal $\mathrm{A} \beta$ and SUVR mean/median. We did not observe any confounding of $\mathrm{wS}_{2}$ by region size or injected dose.

Conclusion: The $\mathrm{wS}_{2}$ detects subtle changes and provides additional information about the binding characteristics of radiotracers and $A \beta$ accumulation that are difficult to verify with mean SUVR alone.

Keywords: amyloid-beta plaques, positron emission tomography, 11C-Pittsburgh compound B, statistical descriptors, two-point correlation function

\section{Introduction}

The presence of amyloid-beta $(A \beta)$ plaques in the cortical gray matter is among the major pathological features of Alzheimer's disease (AD), and is present years before memory decline and other clinical manifestations emerge. ${ }^{1-7}$ Reliable diagnostic and prognostic information in the early stages of AD is necessary for clinical interventions that can halt disease progression. More so, there exists a substantial need for quantitative imaging techniques that can sensitively monitor changes in amyloid status over time and determine the response to therapeutic interventions. A $\beta$ plaques have

Correspondence: Sepideh Shokouhi Vanderbilt University Institute of Imaging Science, II6I 2Ist Avenue South, Medical Center North, AA-I I05, Nashville,

TN 37232-2310, USA

$\mathrm{Tel}+\mathrm{I} 6153226214$

Fax + I 6153220734

Email sepideh.shokouhi@vanderbilt.edu 
been imaged in the human brain using positron emission tomography (PET) with radiotracers, such as 11C-Pittsburgh compound B (11C-PiB $)^{8}$ and 18 F-florbetapir. ${ }^{9}$ In an attempt to quantify levels of in vivo amyloid plaque, contemporary analyses rely on the calculation defined as the ratio of image intensity between the cortex and a reference region (commonly the cerebellar gray matter). This method, the standardized uptake value ratio (SUVR), is appealing due to its computational simplicity and short scan time. Radiotracer uptake is measured after binding in the cortex and reference region reaches a steady state $40-50$ minutes after injection. ${ }^{3}$ Healthy cortex typically shows tracer binding at an SUVR of 1.5 or less. Any ratio above this threshold is referred to as "PiB-positive" and is thought to be indicative of abnormal accumulation of $A \beta$ plaques. Another method employs kinetic modeling of amyloid-PET, and is computed from dynamic data ${ }^{10,11}$ collected over a longer acquisition time, but is more susceptible to subject movement. Both the SUVR and the kinetic modeling methods typically rely on the mean or median of the voxel activity values across a region of interest, but fail to account for variations in the spatial pattern within the region. Consequently, regional SUVR mean values are subject to high variance (large error bars), thus precluding an accurate assessment of longitudinal alterations to $A \beta$ accumulation. Therefore, there exists a need for image analysis methods that can characterize spatial patterns of radiotracer activity in A $\beta$-PET data to provide additional information about longitudinal alteration in distribution of radiotracer activity and $A \beta$ accumulation.

Statistical descriptors such as the two-point correlation function $\left(\mathrm{S}_{2}\right)$ are commonly used in specialties such as material science and astronomy to characterize microscopic properties of materials or galaxy distributions. ${ }^{12-20}$ This metric provides a detailed characterization of spatial patterns in images, typically referred to as clustering or flocculence. Continuousvalued A $\beta$-PET images are analogous to the material science concept of multiphase media where different voxel activity values correspond to different phases. Conventional two-point correlation analysis would require the computation of $\mathrm{S}_{2}$ functions for each voxel value, which is difficult to interpret due to the broad range of activity values within the PET data. In this study, we applied a modified $\mathrm{S}_{2}$ metric, a weighted two-point correlation function $\left(\mathrm{wS}_{2}\right)$, on continuous-valued (nonbinary) 11C-PiB PET to capture longitudinal changes in image spatial patterns with a single statistical function. Here, we introduce a novel computational framework of the $\mathrm{wS}_{2}$ analysis. We then implement this analysis on longitudinal 11C-PiB scans in human subjects and compare the outcomes of $\mathrm{wS}_{2}$ with currently used mean/median SUVR and cerebrospinal fluid (CSF) measures of $\mathrm{A} \beta$.

\section{Material and methods Calculation of two-point correlation function}

Calculation of $\mathrm{S}_{2}$ involves comparison of each voxel with all other voxels within the image. This process can be approximated by selecting $\mathrm{N}$ number of random voxel pairs on a binary image space (voxel values are either 1 or 0 ) with variable intervoxel distances and counting the number of times both voxel values are equal to 1 (true instances). The instances are then binned by the intervoxel distances, and for a given distance $r$, the number of true instances is divided by the total number of instances with distance r. Figure 1 illustrates two binary images with a spatially random distribution of nonzero voxels and their corresponding two-point correlation functions. The two-point correlation function can be interpreted as the probability that two randomly selected image voxels $x_{1}$ and $x_{2}$ both are nonzero. The $x$ axis of the plot in Figure 1 represents the intervoxel distance and the $y$ axis shows the calculated $\mathrm{S}_{2}$ values for the given distance. The two images in Figure 1 are not identical but are statistically similar, thus generating similar $\mathrm{S}_{2}$ curves. Figure 2 shows another set of binary images with the same number of nonzero (black) voxels. While in the first image (Figure 2A) these voxels are randomly distributed, in the second image (Figure 2B) some of them were moved around to form small clusters of 4-5 voxels. The clustering effect changes the $\mathrm{S}_{2}$ function of the second image (dashed line). The area under the curve (AUC) increases with the increase of flocculence (clustering) in the spatial distribution of black voxels. Similarly, more changes in $\mathrm{S}_{2}$ function can be observed when the flocculence increases (Figure 2C). Since all three images have the same fraction of nonzero voxels, their calculated mean voxel values remain unchanged. Therefore, the utilization of the two-point correlation function can reveal additional information about the spatial distribution of the voxel values, which cannot be determined by their calculated mean or median values.

\section{Calculation of weighted two-point correlation function}

Continuous-valued images such as an amyloid-PET can be characterized with multiple two-point correlation functions, one for each voxel value. ${ }^{20}$ However, typical amyloid-PET images have more than a few activity values. Therefore, such an approach would require computation of a large number of $\mathrm{S}_{2}$ functions, which is not only computationally intensive 

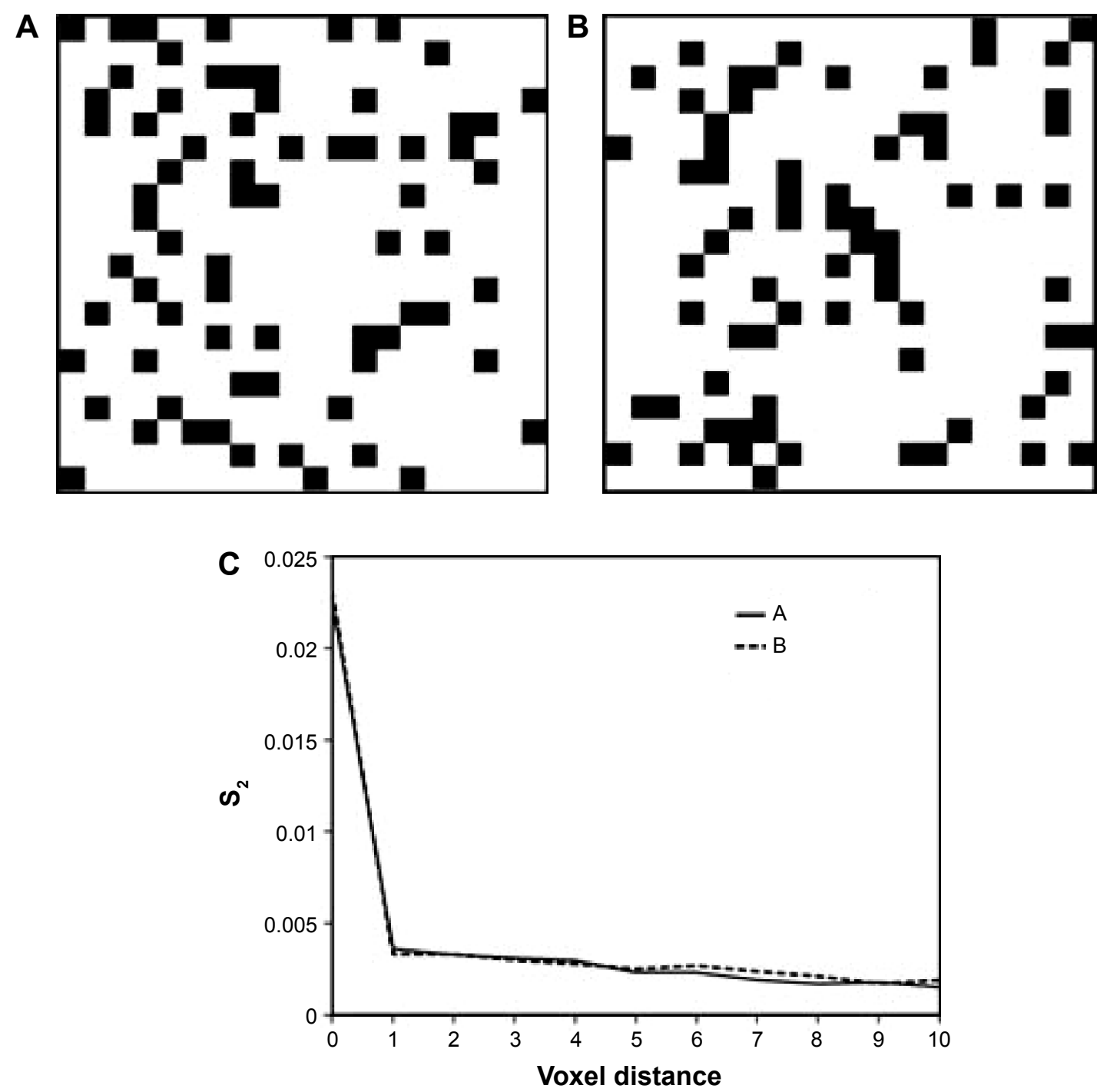

Figure I Two nonidentical binary images (A, B) with similar statistical properties in their nonzero voxel distribution, and their corresponding two-point correlation functions (C). The horizontal axis of the plot in $C$ indicates the distance between voxels, and the vertical axis indicates the probability of finding two black voxels at a given distance. Because there is no spatial clustering in images $\mathbf{A}$ and $\mathbf{B}, \mathbf{S}_{2}$ drops off rapidly as a function of distance in $\mathbf{C}$.

but also the results are difficult to interpret. To address this challenge, we formulated a weighted two-point correlation function $\left(\mathrm{wS}_{2}\right)$ to facilitate implementation of a single statistical function on a nonbinary image. Similar to $\mathrm{S}_{2}, \mathrm{wS}_{2}$ is estimated by randomly selecting $\mathrm{N}$ pairs of voxels at variable distances on the image space. For $\mathrm{S}_{2}$, each instance is weighted either zero (when one or both voxels are zero) or one (when both voxels are nonzero). For $\mathrm{wS}_{2}$, we generalize this by computing a weighting factor that preferentially weights instances where both voxels have high activity. We used the following equation as an initial approach in defining a weighting scheme:

$$
W=\frac{I_{1}+I_{2}}{2} \cdot e^{-\left|\left(I_{1}-I_{2}\right)\right|}
$$

The weighting factor for each instance, $W$, is calculated as the product of two terms. The first term is the average value of the two selected voxels $\left(I_{1}, I_{2}\right)$. The second term incorporates the absolute difference between the two voxel values into an exponential decay. This weighting scheme takes advantage of the information encoded by the image intensities. Figure 3 illustrates the weighting schemes in $\mathrm{S}_{2}$ for binary images and in $\mathrm{wS}_{2}$ for nonbinary images. Figure 4 shows the weighted two-point correlation functions of three simulated nonbinary images. The AUC and the slope changes with the clustered increase of activity from the upper image (Figure 4A) to the lower image (Figure 4C). Implemented on amyloid-PET images, the $\mathrm{wS}_{2}$ can be used to capture the flocculence of the tracer activity within different cortical regions. While several factors, such as the nonspecific 

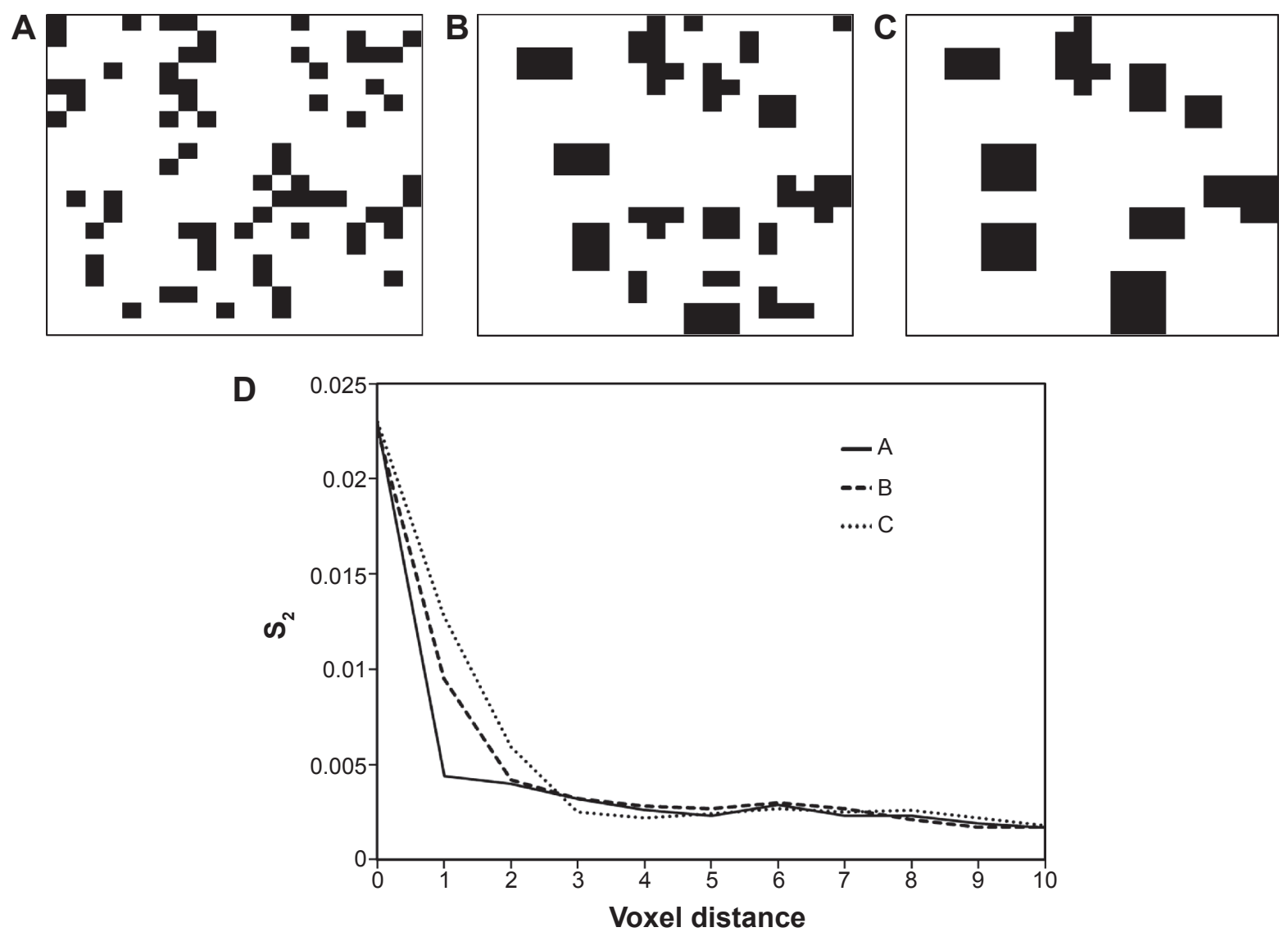

Figure 2 Three binary images with equal fractions of nonzero voxels. In (A) the black voxels are randomly distributed, while in (B) and (C) they are increasingly clustered. (D) The $\mathrm{S}_{2}$ functions of the three images are compared. As the clustering of nonzero voxels increases, the value of $\mathrm{S}_{2}$ at shorter distances increases, as does the area under the curve.

radiotracer binding, partial volume effect, and white matter retention contribute to the activity pattern within a region of a PET image, we hypothesize that the observed clustering of the activity distribution and the spread of high-activity clusters could be partially attributed to underlying heterogeneity in the spatial distribution of $A \beta$ plaques and the specific binding of the radiotracer. The presence of regional activity clusters in longitudinal PET data is often qualitatively visible, as shown in Figure 5, with images of 11C-PiB in four different regions of three Alzheimer's Disease Neuroimaging Initiative (ADNI) subjects. The regional activity distribution of baseline images (Figure 5A, C, E, and G) were compared with their follow-up time points (Figure 5B, D, F, and H). All images show localized patterns of high activity clusters, which seem to remain in the same area within the course of one or 2 years. With the weighted two-point correlation functions, we can define a quantitative metric that characterizes the clustered increase of activity in different regions or in the whole brain. The next sections of this study provide details on 11C-PiB PET acquisition and preprocessing, as well as implementation of $\mathrm{wS}_{2}$ on two-dimensional and three-dimensional PET data and evaluation.

\section{Subjects}

Data used in the preparation of this paper were obtained from the ADNI database (adni.loni.usc.edu). The ADNI was launched in 2003 by the National Institute on Aging, the National Institute of Biomedical Imaging and Bioengineering, the US Food and Drug Administration, private pharmaceutical companies, and non-profit organizations as a $\$ 60$ million, 5-year public-private partnership. The primary goal of the ADNI has been to test whether serial magnetic resonance imaging (MRI), PET, other biological markers, and clinical and neuropsychological assessment can be combined to measure the progression of mild cognitive impairment (MCI) and early AD. Determination of sensitive and specific markers of very early $\mathrm{AD}$ progression is intended to aid researchers and clinicians in the development of new treatments and monitor their effectiveness, as well as lessen the time and cost of clinical trials. The principal investigator of this initiative is Michael W Weiner, from the VA Medical Center and University of California-San Francisco. The ADNI is the result of efforts of many coinvestigators from a broad range of academic institutions and private corporations, and subjects have been recruited from over 50 sites 


\section{$S_{2}$ weighting scheme}

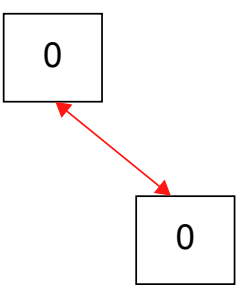

$W=0$

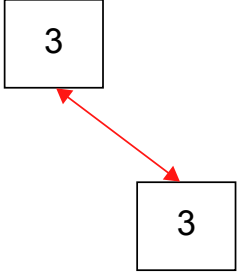

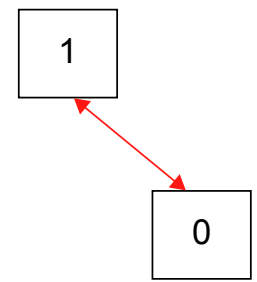

$\mathrm{W}=0$

\section{wS $_{2}$ weighting scheme}
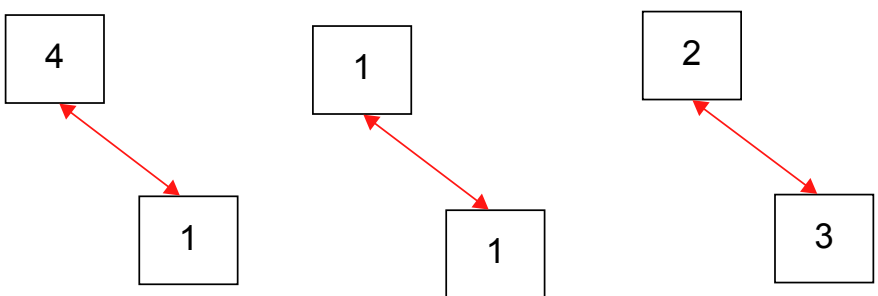

$$
W=\frac{I_{1}+I_{2}}{2} \cdot e^{-\left(I_{1}-I_{2}\right)}
$$

Figure 3 Illustration of weighting schemes in $\mathrm{S}_{2}$ and $\mathrm{wS}_{2}$. The conventional $\mathrm{S}_{2}$ metric utilizes a binary weighting scheme where each event is weighted as either zero (if one or both voxels are zero) or one (if both voxels are nonzero). In wS, we utilize equation I to calculate the weighting factor. The higher and the closer the voxel values, the higher the weighting factor of the event.

across the USA and Canada. The initial goal of the ADNI was to recruit 800 subjects, but the ADNI has been followed by ADNI-GO and ADNI-2. To date, these three protocols have recruited over 1,500 adults aged 55-90 years to participate in the research, consisting of cognitively normal older individuals, people with early or late MCI, and people with early AD. The follow-up duration of each group is specified in the protocols for ADNI-1, ADNI-2, and ADNI-GO. Subjects originally recruited for ADNI-1 and ADNI-GO had the option to be followed in ADNI-2. For up-to-date information, see www.adni-info.org.

We used 31 ADNI subjects from MCI, late mild MCI, and the old healthy control (normal) cohort. Table 1 summarizes the demographic and clinical data of the subjects used in this study. The subjects were selected based on the availability of longitudinal $11 \mathrm{C}-\mathrm{PiB}$ scans. In addition to longitudinal PiB-PET scans, 13 subjects also had longitudinal CSF measures obtained at the same time points as the PiB scans. The ADNI UPENN-Longitudinal-Biomarker Data (4-year) file was searched to identify these subjects, whose PiB-PET data were used for three-dimensional analysis of the whole brain $\mathrm{wS}_{2}$ and its association with CSF $\mathrm{A} \beta$. The remaining subjects who did not have complete biospecimen information were used for two-dimensional $\mathrm{wS}_{2}$ analysis of different brain regions and its association with region size and image noise.

\section{Data acquisition and preprocessing}

11C-PiB PET from baseline and follow-up scans together with T1-weighted MRI volumes were downloaded from the ADNI database. While some subjects had follow-up scans at both 12 months and 24 months after baseline, others had only one follow-up at either 12 months or 24 months. All PiB-PET scans were started 50 minutes after injection, allowing for tracer uptake and nonspecific washout. Four dynamic frames with a scan duration of 20 minutes were coregistered, averaged into a single image, and normalized with respect to cerebellar gray matter. All preprocessing steps were performed by the Laboratory of Neuroimaging at the University of Southern California. We downloaded the preprocessed PET data that were coregistered, averaged, and intensity-normalized to the cerebellar gray matter. 


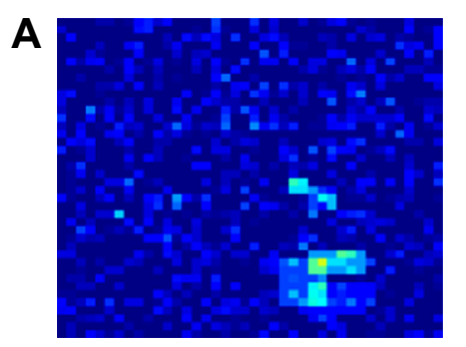

B
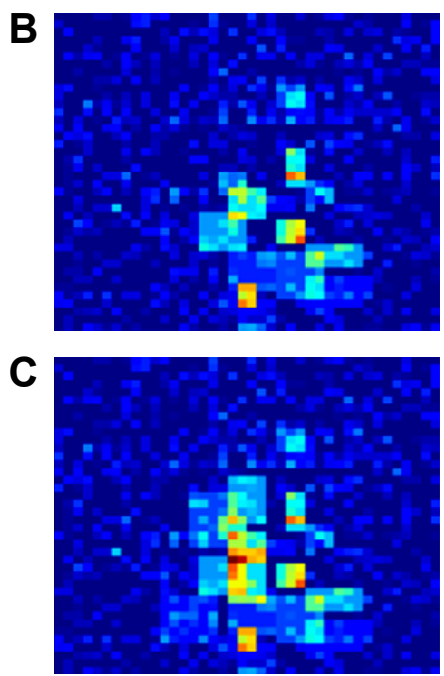

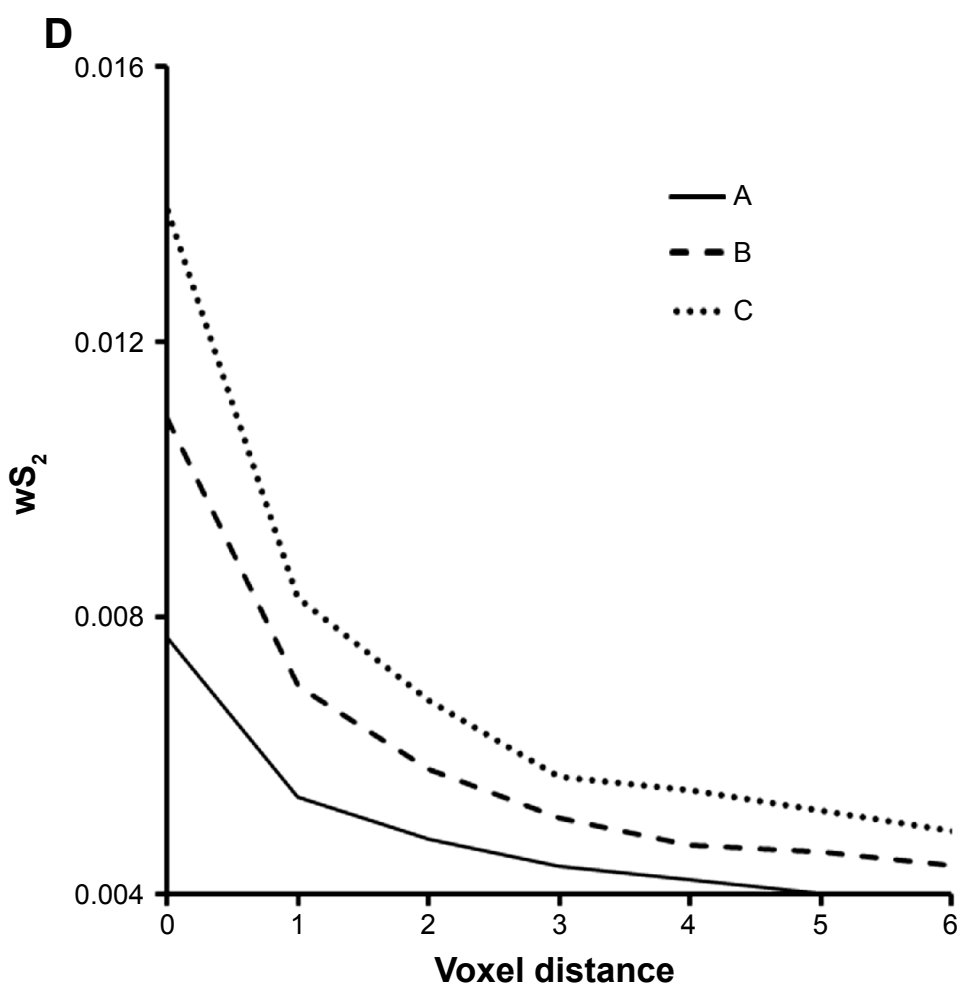

Figure 4 Weighted two-point correlation functions (D) of three simulated continuous-valued (nonbinary) images (A-C).

Note: Area under the curve and its slope change in figure $\mathbf{D}$ with the clustered increase of activity in images $\mathbf{A}, \mathbf{B}$ and $\mathbf{C}$. The image with the smallest cluster (A) corresponds to the function with smallest area under the curve. The image with highest and largest cluster of activity (C) shows the two-point correlation with largest area under the curve.

In addition, we limited our PiB-PET data selection to those scans that were performed with the same scanner, acquisition protocol, and image reconstruction method at baseline and each follow-up to reduce variations due to scanner resolution or sensitivity.

\section{$\mathrm{wS}_{2}$ analysis on two-dimensional data}

For each subject we sampled axial PiB image slices from several different brain regions, including the occipital lobe and the bilateral temporal, parietal, and frontal lobes. The T1-weighted MRI volume was used to define these regions using the methods described by Riddle et al. ${ }^{21}$ Briefly, these anatomical regions were obtained by aligning the subject's T1-weighted MRI volume (acquired at a time point close to the PiB PET baseline scan) with the T1-weighted MRI volume from a reference brain where the regions were segmented manually. The deformation field between the reference brain and the subject's T1-weighted MRI volume was calculated with a nonrigid demons registration algorithm. ${ }^{22}$ The regional binary masks from the reference brain were warped to the subject's image volume with the nonrigid deformation field. Each brain volume was also segmented into CSF, gray matter, and white matter with Fuzzy C-means ${ }^{23}$ and a gray matter mask was created for each anatomical region. These regional gray matter masks were applied to the PiB-PET images that were coregistered to the subject's MRI volume in SPM8 (http://www.fil.ion.ucl.ac.uk/spm/). Altogether, we had over 300 sets of two-dimensional images that served as starting points for our two-dimensional analysis, which aimed to investigate the association between SUVR and $\mathrm{wS}_{2}$ in different regions and to determine whether the $\mathrm{wS}_{2}$ outcomes were biased by the region size, which was defined as the number of gray matter voxels within a region where the $\mathrm{wS}_{2}$ voxel pairs were sampled from. For the $\mathrm{wS}_{2}$ calculation, we generated 100,000 random voxel pairs located within the gray matter of that region. All instances were weighted using equation 1, binned by the Euclidean voxel distances $r$ between zero and $10 \mathrm{~mm}$, and divided by the number of instances for each distance to obtain the $\mathrm{wS}_{2}(\mathrm{r})$. The error associated with the $\mathrm{wS}_{2}$ curves was calculated by resampling 20 ensembles of 100,000 voxel pairs and calculating the standard deviation. We found that additional resampling beyond 20 did not increase the error bars. The areas under the $\mathrm{wS}_{2}$ curves (AUC) from zero to $10 \mathrm{~mm}$ distance were used as quantitative values to characterize the increased clustered activity and how it related to changes in SUVR mean values. 

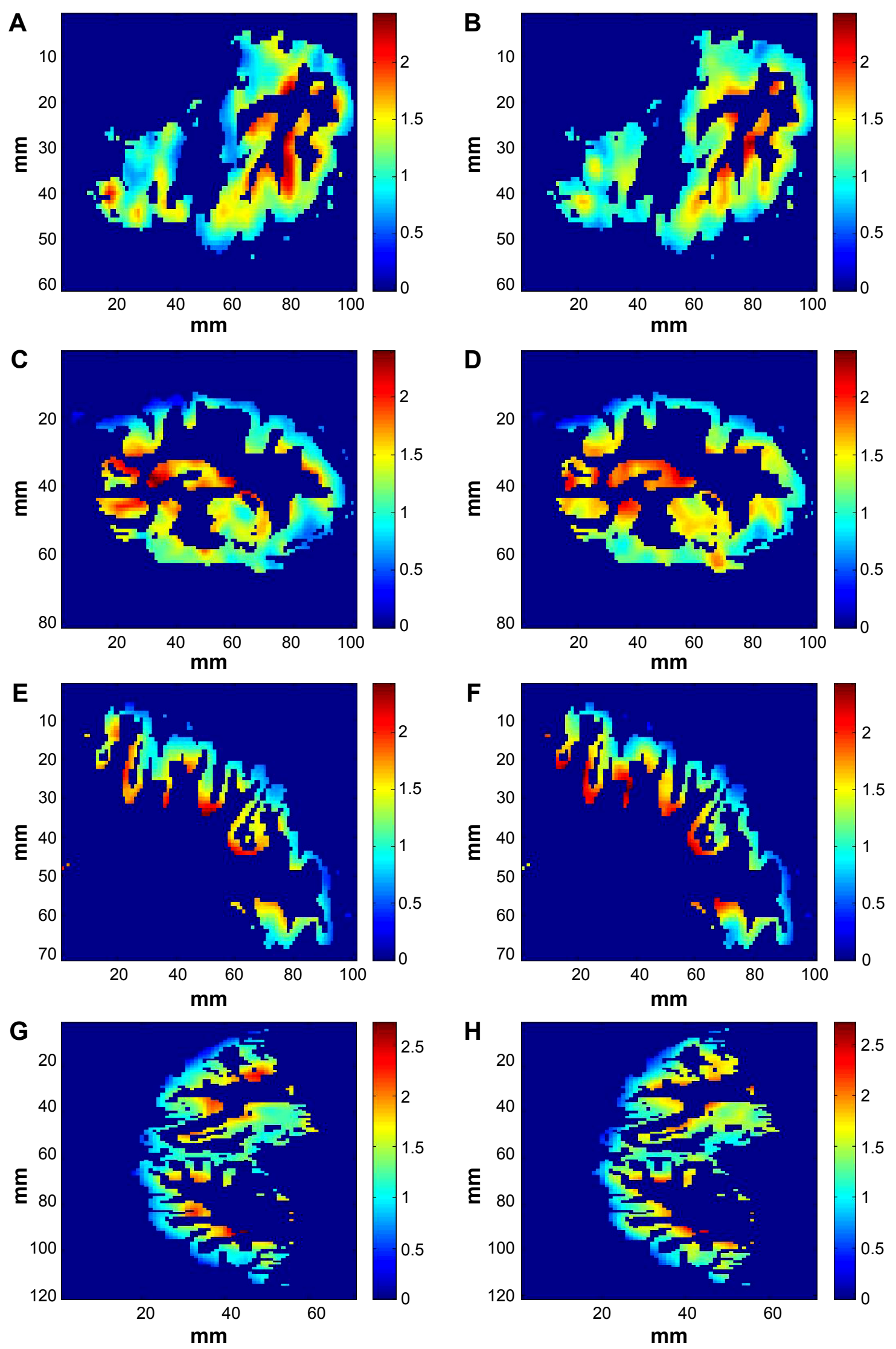

Figure 5 Four sample images of I IC-Pittsburgh compound B in four different regions of three subjects from the Alzheimer's Disease Neuroimaging Initiative. The regional activity distribution of the baseline images (A, C, E, G) are compared with their follow-up time points (B, D, F, H). There is a clearly noticeable localized pattern of clustered activity, which seems to remain in the same area and is slightly spread within the course of I2-24 months. (A, B) Right temporal lobe in subject 010_S_04I29. (B, C) Left temporal lobe in subject 005_S_0546. (D, E) Left frontal lobe in subject 04I_S_0898. (G, H) Occipital lobe in subject 04I_S_0898. 
Table I Clinical data for Alzheimer's Disease Neuroimaging Initiative subjects used for this study

\begin{tabular}{llll}
\hline & Total & Female & Male \\
\hline Number of subjects & 31 & 13 & 18 \\
Baseline age, $y$ & $76 \pm 7$ & $76 \pm 8$ & $77 \pm 6$ \\
Late MCl, $\mathrm{n}$ & 15 & 7 & 8 \\
$\mathrm{MCl}, \mathrm{n}$ & 7 & 1 & 6 \\
Normal, $\mathrm{n}$ & 9 & 5 & 4 \\
APOE AI/A2 carrier, $\mathrm{n}$ & 17 & 7 & 10 \\
\hline
\end{tabular}

Abbreviations: $\mathrm{APOE}$, apolipoprotein $\mathrm{E} ; \mathrm{MCl}$, mild cognitive impairment; $y$, years.

We implemented a linear mixed-effects model to determine the effect of the region size on $\mathrm{wS}_{2}$. We also utilized another statistical analysis to determine whether longitudinal changes in the regional $\mathrm{wS}_{2}$ curves were affected by differences in the administered radiotracer dose at baseline and follow-up time points. This was analyzed with mixed-effects models using the R software package (www.r-project.org). The AUC at the follow-up time points was predicted by baseline (time 0 ) AUC, time point (1 or 2 ), region size, and change in injection dose relative to baseline as fixed effects.

\section{Three-dimensional $\mathrm{wS}_{2}$ analysis of whole brain}

The whole brain PiB-PET images were aligned to the T1-weighted MRI image at a time point closest to the baseline PET. The gray matter mask of the whole brain T1-weighted MRI was overlaid with the PiB-PET. We generated 50,000 random voxel pairs located within the gray matter fraction of the PET images to calculate the $\mathrm{wS}_{2}$. The AUCs from zero to $15 \mathrm{~mm}$ distance were calculated and compared with the CSF measures obtained from the ADNI database. For the CSF measures, lumbar punctures were performed at the participating ADNI sites and are described in the ADNI biospecimen protocols (adni.loni. ucla.edu/research/protocols/biospecimens-protocols). The CSF $\mathrm{A} \beta_{1-42}$ was obtained from a multiplex xMAP Luminex platform using Innogenetics immunoassay kit-based reagent. Using the Spearman's rank correlation, we calculated the correlation coefficients between the AUC of the $\mathrm{wS}_{2}$ curves and $1 / A \beta$. There is an inverse correlation between CSF A $\beta$ and PET images. For convenience, we used $1 / A \beta$ instead of $A \beta$ to take away the negative sign from the correlation coefficient. Similarly, we calculated the correlation coefficients between the whole brain PiB-PET SUVR mean and median values and the CSF $A \beta$ measures. In addition to Spearman's correlation, we utilized a linear mixed-effects (AR1) model to evaluate temporal correlations between $\mathrm{wS}_{2}$, SUVR mean and SUVR median (outcome variables) and CSF $A \beta$ (predictor).

\section{Results}

\section{$\mathrm{wS}_{2}$ analysis on two-dimensional data}

The implementation of $\mathrm{wS}_{2}$ on two-dimensional images not only offered a visual comparison of this method with the observed qualitative changes in the PET data, but also allowed a comparison between $\mathrm{wS}_{2}$ and SUVR mean values in different brain regions. Figure 6 shows a sample 11C-PiB image from a subject's right temporal lobe at baseline (Figure 6A) and follow-up (Figure 6B). Figure 6C shows the calculated SUVR mean values of the images at baseline and follow-up, and Figure 6D shows their corresponding $\mathrm{wS}_{2}$ curves. Both the SUVR mean and the $\mathrm{wS}_{2}$ method indicate little change between baseline and follow-up. For comparison, Figure 7 shows the 11C-PiB images from another subject's left parietal lobe at baseline (Figure 7A) and follow-up (Figure 7B). Figure 7C shows the calculated SUVR mean values at baseline and follow-up. Due to the large error bars, it is difficult to determine whether there is a significant change in the SUVR mean. In comparison with the SUVR mean value, the $\mathrm{wS}_{2}$ curves at the two time points (Figure 7D) are clearly separated. For the group level, the Spearman's rank correlation between the $\mathrm{wS}_{2}$ and SUVR mean is 0.9 with $P<0.001$. We used a linear mixed-effects model analysis to determine whether longitudinal changes in the $\mathrm{wS}_{2}$ curves were affected by region size or differences in the radiotracer dose administered at baseline and follow-up time points. The outcomes of this analysis are summarized in Table 2. For the effect of region size on AUC, we obtained a $P$-value of 0.61 , indicating that the size of the region from where the voxel pair was sampled does not affect the $\mathrm{wS}_{2}$ outcomes. For the effect of differences in injected dose between the baseline and follow-up scan, we obtained a $P$-value of 0.28 , indicating no significant effect.

\section{Three-dimensional $\mathrm{wS}_{2}$ analysis on whole brain}

The visit-to-visit association between the whole brain AUCs of $\mathrm{wS}_{2}$ and the CSF $1 / \mathrm{A} \beta$ were calculated using the Spearman's correlation coefficient. The outcomes were compared with the correlation between the SUVR mean/median values and CSF measures at baseline and 12-month follow-up. All correlation outcomes are summarized in Table 3 . We found a slightly higher inverse correlation between $\mathrm{wS}_{2}$ and CSF $\mathrm{A} \beta$. These were 0.82 for baseline and did not change significantly (0.8) after 12 months. For comparison, the correlation between the SUVR mean and CSF A $\beta$ decreased from 0.83 to 0.6 after 12 months. Similarly, the correlation between SUVR median value and CSF $A \beta$ decreased from 0.83 to 0.6. We also utilized a linear mixed-effects (AR1) model as an 

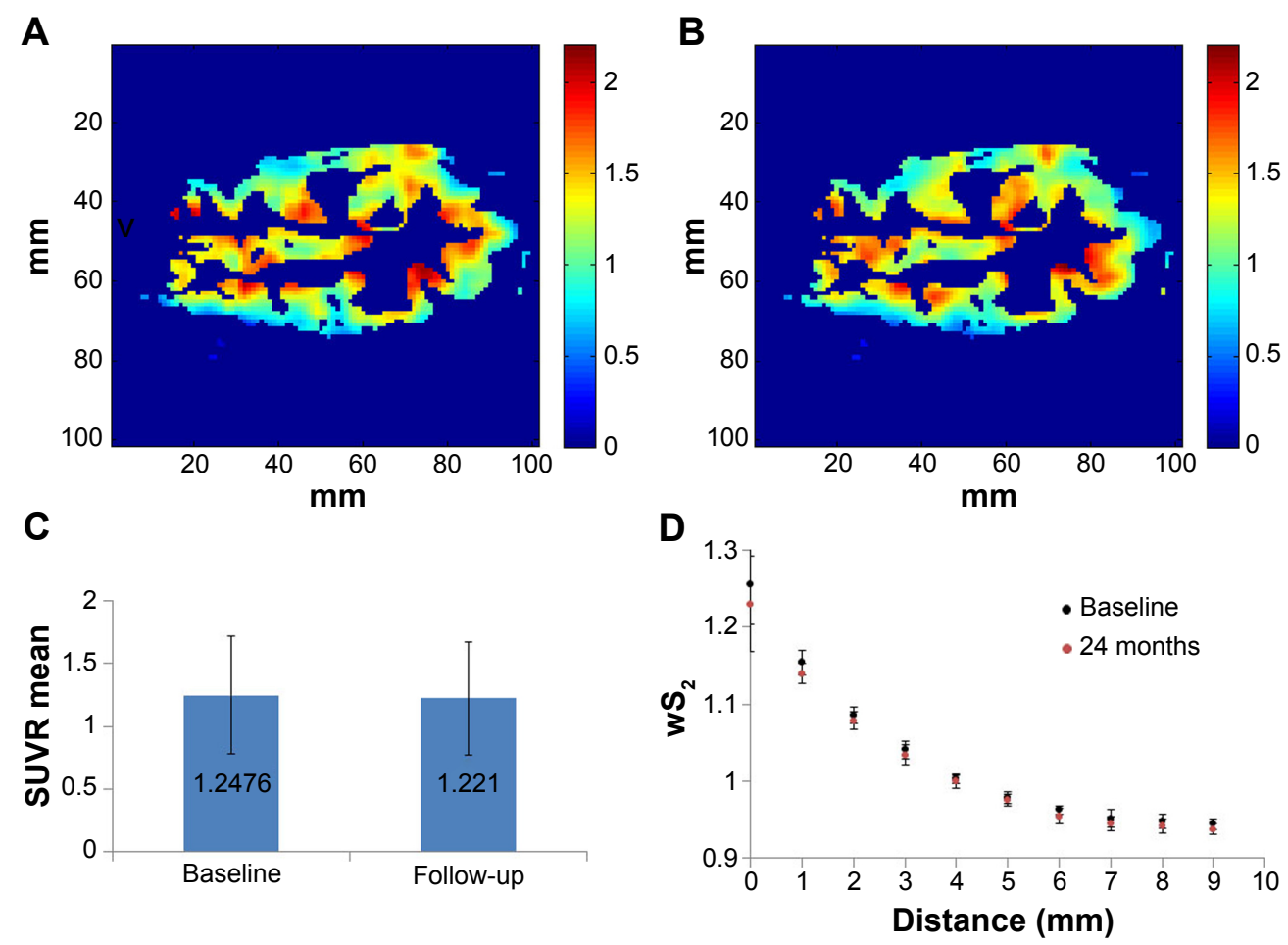

Figure 6 I IC-Pittsburgh compound B image from the right temporal lobe at baseline (A) and follow-up (B) positron emission tomography scan. Mean standardized uptake value ratio values for images $(\mathbf{C})$ at baseline and (D) follow-up showing their $\mathrm{wS}_{2}$ curves.

Abbreviation: SUVR, standardized uptake value ratio.

A

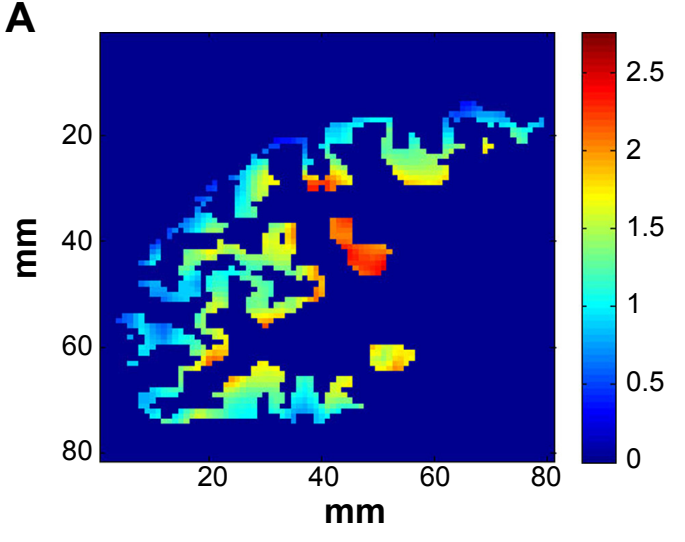

C

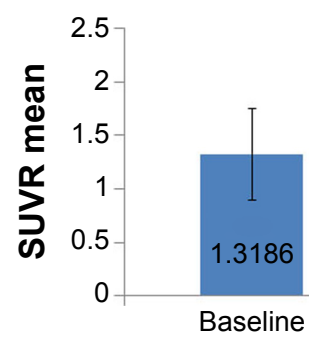

B

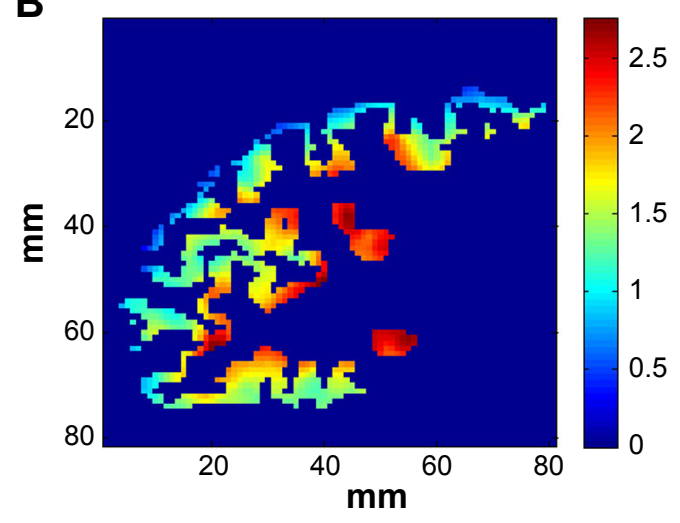

D

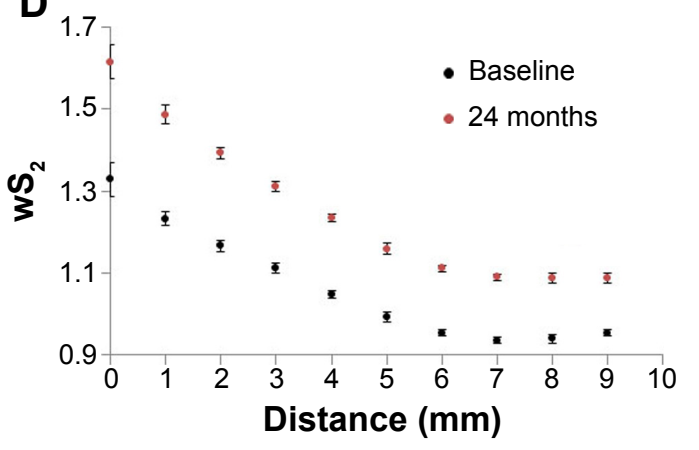

Figure 7 IIC-Pittsburgh compound B image from the left parietal lobe at baseline (A) and follow-up (B) positron emission tomographic scan. (C) Mean standardized uptake value ratio values for images at baseline and follow-up and (D) their $\mathrm{wS}_{2}$ curves.

Abbreviation: SUVR, standardized uptake value ratio. 
Table 2 Outcome results for the two mixed-effects models investigating the effect of region size (number of gray matter voxels) and difference between baseline and follow-up injected dose (noise mismatch) on changes in the AUC

\begin{tabular}{ll}
\hline AUC & \\
\hline Region size $\left(\mathrm{AUC} / \mathrm{mm}^{3}\right)$ & 0.13 \\
$95 \% \mathrm{Cl}$ & $-0.38,0.63$ \\
$P$-value & 0.61 \\
Delta injection dose $(\mathrm{AUC} / \mathrm{mCi})$ & 0.06 \\
$95 \% \mathrm{Cl}$ & $-0.05,0.17$ \\
$P$-value & 0.28 \\
\hline
\end{tabular}

Abbreviations: AUC, area under the curve; $\mathrm{Cl}$, confidence interval.

additional method to evaluate temporal correlations between $\mathrm{wS}_{2}$, SUVR mean and SUVR median (outcome variables) and CSF A $\beta$ (predictor). Based on this model, we observed similarly significant inverse correlation between CSF A $\beta$ and all three brain PET measures. These were $0.45(P=0.005)$ for $\mathrm{wS}_{2}, 0.45(P=0.008)$ for SUVR mean, and $0.39(P=0.02)$ for SUVR median.

\section{Discussion}

This is the first study that implements two-point correlation functions to detect longitudinal changes in A $\beta$-PET data. We formulated a weighted two-point correlation to characterize the clustered activity increase of 11C-PiB PET images and its association with CSF outcomes and SUVR mean values. We used a weighting function that preferentially weights instances where both voxels have high activity as an initial approach for implementing a single correlation function on continuous-valued amyloid-PET data. All PET data were normalized to the cerebellum gray matter, allowing utilization of a simple weighting scheme for $\mathrm{wS}_{2}$. The objective of this study was to validate the $\mathrm{wS}_{2}$ technique. In the future, we will implement this method on amyloidPET data that are not normalized by the cerebellum or any other reference region, thus minimizing potential errors associated with reference tissue normalizations. The $\mathrm{wS}_{2}$

Table 3 Spearman's rank correlation coefficients and $P$-values between the AUC/SUVR mean/SUVR median and I/A $\beta$ in cerebrospinal fluid

\begin{tabular}{|c|c|c|c|c|}
\hline & \multicolumn{2}{|l|}{$I / A \beta$} & \multicolumn{2}{|l|}{$I / A \beta$} \\
\hline & Baseline & $P$-value & $12 \mathrm{~m}$ & $P$-value \\
\hline $\mathrm{wS}_{2}$ & 0.82 & 0.007 & 0.8 & 0.003 \\
\hline Mean & 0.83 & 0.005 & 0.6 & 0.04 \\
\hline Median & 0.83 & 0.005 & 0.6 & 0.04 \\
\hline
\end{tabular}

Abbreviations: AUC, area under the curve; SUVR, standardized uptake value ratio. approach on non-normalized PET data would require more thoroughly evaluated weighting functions, which we intend to explore further. We used the area under the $\mathrm{wS}_{2}$ function as a quantitative outcome of this method. Other characteristic features, such as the shape or the temporal slope of the $\mathrm{wS}_{2}$ function, may be used as additional quantitative parameters to characterize longitudinal changes, particularly for nonnormalized amyloid-PET data. Similar to the SUVR mean analysis, the $\mathrm{wS}_{2}$ approach can be performed on static PET scans and does not require long dynamic acquisitions. The $\mathrm{wS}_{2}$ metric has some characteristics that offer potential advantages over the conventional typical SUVR mean value. It is associated with smaller variance (error bars), which is particularly useful for clinicians who are interested in detecting small changes in longitudinal amyloid-PET as a response to therapeutic interventions. The large error bars of the SUVR mean value often make it difficult to detect subtle changes, and the $\mathrm{wS}_{2}$ analysis can provide additional information to assess for possible alterations. Implemented on an $\mathrm{AD}$ cohort, the two-point correlation function could be used to determine whether amyloid accumulation remains static in the AD phase. The $\mathrm{wS}_{2}$ method could be also utilized as a useful tool to compare the regional patterns of activity distribution between different amyloid-PET radiotracers in the same subject and gain more insight into differences in radiotracer binding characteristics. As the $P$-values in Table 2 indicate, we did not see any significant effect on AUC related to region size or dose injected. The injected dose was used as a surrogate for image noise to determine whether possible noise mismatches between baseline and follow-up scans would be considered as confounding factors in the $\mathrm{wS}_{2}$ analysis. While differences in scanner sensitivity also contribute to the noise, we were able to disregard the scanner effect by selecting PiB-PET data that were performed with the same scanner at baseline and follow-up. The objective of our three-dimensional whole brain $\mathrm{wS}_{2}$ analysis was to compare the AUC outcomes with the CSF measures of $A \beta_{1-42}$, which are known biomarkers of AD pathology. The decrease in CSF $A \beta_{1-42}$ is associated with increased activity in $11 \mathrm{C}-\mathrm{PiB}$ PET images, as investigated by several previous studies. ${ }^{24-28}$ The correlation coefficients between the SUVR mean and the CSF $A \beta_{1-42}$ from our study were also comparable with those in a previous study by Forsberg et $\mathrm{al}^{26}$ investigating the relationship between $\mathrm{PiB}$ retention and CSF A $\beta_{1-42}$ in an $\mathrm{MCI}$ cohort. Comparing the $\mathrm{wS}_{2}$ method with the conventional SUVR mean and median values (Table 2), we were able to show a slightly higher inverse correlation between the PiB 
imaging data and CSF A $\beta$. Overall, the $\mathrm{wS}_{2}$ technique can detect subtle changes in the spatial pattern of PiB-PET and efficiently reduce the complexity of implementing statistical descriptors on continuous-valued images. The weighed twopoint correlation technique can be easily adapted for other imaging modalities, such as computed tomography or MRI for a wide range of applications in medicine and biology.

\section{Acknowledgments}

Data collection and sharing for this project was funded by the Alzheimer's Disease Neuroimaging Initiative (ADNI) via the National Institutes of Health (grant U01 AG024904) and Department of Defense (award W81XWH-12-2-0012). The ADNI is funded by the National Institute on Aging, the National Institute of Biomedical Imaging and Bioengineering, and through generous contributions from the Alzheimer's Association, Alzheimer's Drug Discovery Foundation, Araclon Biotech, BioClinica, Inc, Biogen Idec Inc, Bristol-Myers Squibb Company, Eisai Inc, Elan Pharmaceuticals, Inc, Eli Lilly and Company, EuroImmun, F. Hoffmann-La Roche Ltd and its affiliated company Genentech, Inc, Fujirebio, GE Healthcare, IXICO Ltd, Janssen Alzheimer Immunotherapy Research and Development, LLC, Johnson \& Johnson Pharmaceutical Research and Development LLC, Medpace, Inc, Merck \& Co., Inc, Meso Scale Diagnostics, LLC, NeuroRx Research, Neurotrack Technologies, Novartis Pharmaceuticals Corporation, Pfizer Inc, Piramal Imaging, Servier, Synarc Inc, and Takeda Pharmaceutical Company. The Canadian Institutes of Health Research is providing funds to support ADNI clinical sites in Canada. Private sector contributions are facilitated by the Foundation for the National Institutes of Health (www. fnih.org). The grantee organization is the Northern California Institute for Research and Education, and the study is coordinated by the Alzheimer's Disease Cooperative Study at the University of California, San Diego. ADNI data are disseminated by the Laboratory for Neuro Imaging at the University of Southern California. This study was supported by NIH/NIBIB grants R00 EB 009106 (to SS) and K23 NS080988 (to DC).

The authors would like to thank John Gore, Todd Peterson, Noor Tantawy, Harrison Barrett, Antonio Sastre, Salvatore Torquato, Andreas Berlind, Kristen Nelson, and Philip Crooke for their supportive discussions, Lucy Qu and Hyeyon Kim for helping with the data analysis, and Benoit M Dawant and Rui Li for the providing the rigid and demons registration software.
Data used in preparation of this article were obtained from the ADNI database (adni.loni.usc.edu). As such, the investigators within the ADNI contributed to the design and implementation of ADNI and/or provided data but did not participate in analysis or writing of this report. A complete listing of ADNI investigators can be found at: http://adni.loni.usc.edu/wp- content/uploads/how to apply/ ADNI Acknowledgement List.pdf.

\section{Disclosure}

The authors report no conflicts of interest in this work.

\section{References}

1. Hardy J, Selkoe DJ. The amyloid hypothesis of Alzheimer's disease: progress and problems on the road to therapeutics. Science. 2002;297: 353-356.

2. McKhann GM, Knopman DS, Chertkow H, et al. The diagnosis of dementia due to Alzheimer's disease: recommendations from the National Institute on Aging-Alzheimer's Association workgroups on diagnostic guidelines for Alzheimer's disease. Alzheimers Dement. 2011;7:263-269.

3. Rowe CC, Ng S, Ackermann U, et al. Imaging beta-amyloid burden in aging and dementia. Neurology. 2007;68:1718-1725.

4. Rowe CC, Ellis KA, Rimajova M, et al. Amyloid imaging results from the Australian Imaging, Biomarkers and Lifestyle (AIBL) study of aging. Neurobiol Aging. 2010;31:1275-1283.

5. Morris JC, Roe CM, Grant EA, et al. Pittsburgh compound B imaging and prediction of progression from cognitive normality to symptomatic Alzheimer disease. Arch Neurol. 2009;66:1469-1475.

6. Thal DR, Rüb U, Orantes M, Braak H. Phases of A beta-deposition in the human brain and its relevance for the development of AD. Neurology. 2002;58:1791-1800.

7. Rabinovici GD, Rosen HJ, Alkalay A, et al. Amyloid vs FDG-PET in the differential diagnosis of AD and FTLD. Neurology. 2011;77: 2034-2042.

8. Klunk WE, Engler H, Nordberg A, et al. Imaging brain amyloid in Alzheimer's disease with Pittsburgh compound-B. Ann Neurol. 2004; 55:306-319.

9. Choi SR, Golding G, Zhuang ZP, et al. Preclinical properties of 18F-AV-45: a PET agent for A $\beta$ plaques in the brain. $J$ Nucl Med. 2009;50:1887-1894.

10. Logan J, Fowler JS, Volkow ND, Wang GJ, Ding YS, Alexoff DL. Distribution volume ratios without blood sampling from graphical analysis of PET data. J Cereb Blood Flow Metab. 1996;16:834-840.

11. Price JC, Klunk WE, Lopresti BJ, et al. Kinetic modeling of amyloid binding in humans using PET imaging and Pittsburgh compound-B. J Cereb Blood Flow Metab. 2005;25:1528-1547.

12. Torquato S. Random Heterogeneous Materials: Microstructure and Macroscopic Properties. New York, NY, USA: Springer-Verlag; 2002.

13. Yeong CL, Torquato S. Reconstructing random media. Phys Rev E Stat Nonlin Soft Matter Phys. 1998;57:495.

14. Jiao Y, Stillinger FH, Torquato S. Modelling heterogeneous material via two-point correlation functions: basic principles. Phys Rev E Stat Nonlin Soft Matter Phys. 2007;76:031110.

15. Debye P, Anderson HR. The correlations function and its application. J Appl Phys. 1957;28:679.

16. Peebles PJE. The Large Scale Structure of the Universe. Princeton, NJ, USA: Princeton University Press; 1980.

17. Rivolo AR. The two-point galaxy correlation function of the Local Supercluster. Astrophys J. 1986;301:70-76. 
18. Dalton GB, Efstathiou G, Sutherland WJ, Maddox SJ, Davis M. The two-point correlation function of rich clusters of galaxies: results from an extended APM cluster redshift survey. Mon Not $R$ Astron Soc. 1994;271:L47.

19. Blair SC, Berge PA, Berryman JG. Using two-point correlation functions to characterize microgeometry and estimate permeabilities of sandstones and porous glass. J Geophys Res. 1996;101(B9):20359-20375.

20. Cooper L, Saltz J, Machiraju R, Huang K. Two-point correlation as a feature for histology images: feature space structure and correlation updating. Conf Comput Vis Pattern Recognit Workshops. 2010:79-86.

21. Riddle WR, DonLevy SC, Wushensky CA, Dawant BM, Fitzpatrick JN, Price RR. Quantifying cerebral changes in adolescence with MRI and deformation based morphometry. J Magn Reson Imaging. 2008;28: 320-326.

22. Dawant BM, Hartmann SL, Thirion JP, Maes F, Vandermeulen D, Demaerel P. Automatic 3-D segmentation of internal structures of the head in MR images using a combination of similarity and free-form transformations. Part I, Methodology and validation on normal subjects. IEEE Trans Med Imaging. 1999;18:909-916.
23. Pham DL, Prince JL. Adaptive fuzzy segmentation of magnetic resonance images. IEEE Trans Med Imaging. 1999;18:737-752.

24. Tolboom N, van der Flier WM, Yaqub M, et al. Relationship of cerebrospinal fluid markers to $11 \mathrm{C}-\mathrm{PiB}$ and $18 \mathrm{~F}-\mathrm{FDDNP}$ binding. $\mathrm{J} \mathrm{Nucl}$ Med. 2009;50:1464-1470.

25. Landau SM, Lu M, Joshi AD, et al; Alzheimer's Disease Neuroimaging Initiative. Comparing positron emission tomography imaging and cerebrospinal fluid measurements of $\beta$-amyloid. Ann Neurol. 2013;74:826-836

26. Forsberg A, Engler H, Almkvist O, et al. PET imaging of amyloid deposition in patients with mild cognitive impairment. Neurobiol Aging. 2008;29:1456-1465.

27. Fagan AM, Mintun MA, Mach RH, et al. Inverse relation between in vivo amyloid imaging load and cerebrospinal fluid Abeta42 in humans. Ann Neurol. 2006;59:512-519.

28. Koivunen J, Pirttilä T, Kemppainen N, et al. PET amyloid ligand [11C] $\mathrm{PiB}$ uptake and cerebrospinal fluid beta-amyloid in mild cognitive impairment. Dement Geriatr Cogn Disord. 2008;26:378-383.
Clinical Interventions in Aging

\section{Publish your work in this journal}

Clinical Interventions in Aging is an international, peer-reviewed journal focusing on evidence-based reports on the value or lack thereof of treatments intended to prevent or delay the onset of maladaptive correlates of aging in human beings. This journal is indexed on PubMed Central, MedLine,

\section{Dovepress}

CAS, Scopus and the Elsevier Bibliographic databases. The manuscript management system is completely online and includes a very quick and fair peer-review system, which is all easy to use. Visit http://www.dovepress. com/testimonials.php to read real quotes from published authors. 